\title{
Hartree simulations of coupled quantum Hall edge states in corner-overgrown heterostructures
}

\author{
L. Steinke, ${ }^{1,2}$ P. Cantwell, ${ }^{3}$ E. Stach, ${ }^{3, *}$ D. Schuh,,${ }^{1, \dagger}$ A. Fontcuberta i Morral, ${ }^{1,4}$ M. Bichler, ${ }^{1}$ G. Abstreiter, ${ }^{1}$ and M. Grayson ${ }^{1,5, \ddagger}$ \\ ${ }^{1}$ Walter Schottky Institut, Technische Universität München, D-85748 Garching, Germany \\ ${ }^{2}$ Max Planck Institute for Chemical Physics of Solids, D-01187 Dresden, Germany \\ ${ }^{3}$ School of Materials Engineering, Purdue University, West Lafayette, Indiana 47907, USA \\ ${ }^{4}$ Laboratoire des Matériaux Semiconducteurs, Institut des Matériaux, EPFL, CH-1015 Lausanne, Switzerland \\ ${ }^{5}$ Department of Electrical Engineering and Computer Science, Northwestern University, Evanston, Illinois 60208, USA
}

(Received 3 August 2012; published 17 April 2013)

\begin{abstract}
The electronic states in a corner-overgrown bent GaAs/AlGaAs quantum well heterostructure are studied with numerical Hartree simulations. Transmission electron microscope pictures of the junction sharpness are shown to justify the sharp-corner potential assumed for these calculations. In a tilted magnetic field, both facets of the bent quantum well are brought to a quantum Hall $(\mathrm{QH})$ state, and the corner hosts an unconventional hybrid system of coupled counter-propagating quantum Hall edges and an additional 1D accumulation wire. We show how, in contrast to coplanar barrier-junctions of QH systems, the coupling between the three subsystems increases as a function of the applied magnetic field, and discuss the implications of the numerical results for the interpretation of experimental data on bent quantum Hall systems reported elsewhere.
\end{abstract}

DOI: 10.1103/PhysRevB.87.165428

PACS number(s): 73.43.-f, 72.20.Ee, 73.63.Nm

\section{INTRODUCTION}

Two-dimensional (2D) and one-dimensional (1D) quantum structures form the cornerstone of scientific discoveries of the past decades from graphene ${ }^{1,2}$ to nanotubes, ${ }^{3}$ from semiconductor quantum wells ${ }^{4-7}$ to quantum wires. ${ }^{8}$ Recent work has shown how quantum wires can also be found in the edges of quantum wells in nanowires. ${ }^{9-11}$ In some cases, the $2 \mathrm{D}$ and $1 \mathrm{D}$ systems can be coupled together in co-planar fashion ${ }^{12}$ or in edge-defined wires. ${ }^{13,14}$ In all cases, magnetic fields can be used to probe the electronic structure and affect the 2D-1D coupling. However, rarely are non-co-planar transport structures manufactured since standard epitaxial fabrication and lithography methods preclude such geometries.

The corner overgrowth technique, ${ }^{15}$ on the other hand, realizes GaAs/AlGaAs heterostructures with two-dimensional (2D) layers bent at a sharp $90^{\circ}$ angle. Magnetotransport measurements on a corner-overgrown bent GaAs/AlGaAs heterointerface structure ${ }^{16-18}$ have already demonstrated the high quality of these samples, showing electron mobilities of up to $2 \times 10^{6} \mathrm{~cm}^{2} /$ Vs at densities of order $10^{11} / \mathrm{cm}^{2}$. In a magnetic field $B$, these systems realize a unique sort of quantum Hall $(\mathrm{QH})$ effect boundary state with either coor counter-propagating one-dimensional (1D) edge modes coupled along the entire corner junction, at filling factors which depend on $B$-field tilt angle. ${ }^{18}$ At equal filling factors $v$ on both facets, the 1D conductance along the corner junction exhibits insulating, weakly insulating, or metallic behavior, depending on $v$, and the metallic behavior represents a realization of the Kane-Fisher disordered antiwire geometry for the fractional quantum Hall effect. ${ }^{16,19,20}$

Here we present numerical Hartree simulations of the band structure and carrier concentrations for bent quantum wells relevant to the transport samples of Refs. 16-18. The sharp confinement potential of the bent quantum well, which is essential to understanding the electronic structure of the device, is demonstrated here by transmission electron microscopy (TEM) measurements of an actual transport sample. The measurements show a corner profile of nanometer-scale sharpness similar to the diagnostic samples studied in Ref. 21. Hartree simulations of these heterostructures are helpful for understanding the electronic states that exist in such nonplanar junctions of two quantum wells and help to interpret their
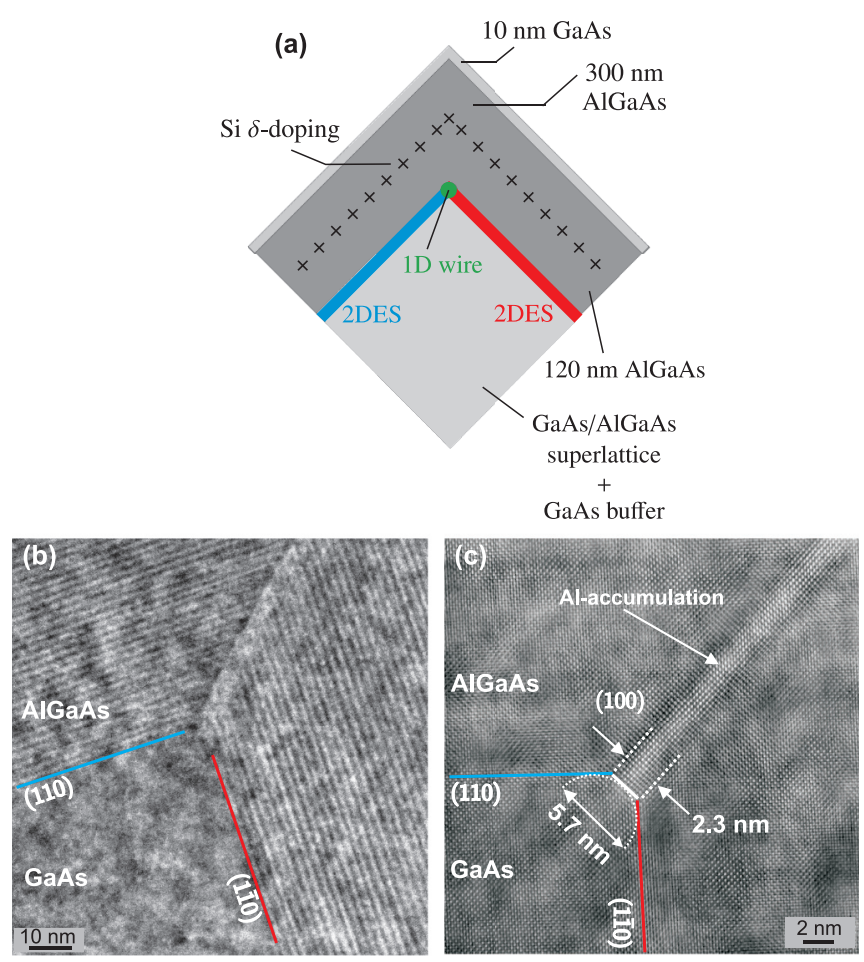

FIG. 1. (Color online) (a) Schematic of the bent quantum well heterostructure. Electrons from the $\mathrm{Si}$ donors in the $\delta$-doping layer accumulate at the interface between the MBE-grown GaAs base layer and the AlGaAs spacer and form 2DES on the two facets (blue and red). At the corner, an additional 1D charge accumulation arises (green). Bright field TEM (a) and phase contrast high-resolution TEM (b) show a cross-section of the bent heterointerface between the GaAs and $\mathrm{Al}_{0.3} \mathrm{Ga}_{0.7}$ As layer. Brighter areas correspond to a higher $\mathrm{Al}$ content per period of rotated growth (see Ref. 21). 
unconventional transport properties. Self-consistent Hartree simulations at zero magnetic field can distinguish regions of charge accumulation or depletion, while spin-degenerate single-electron solutions to the finite $B$ Schroedinger equation provide an elementary understanding of the quantum mechanical states in nonplanar coupled systems of quantum Hall $(\mathrm{QH})$ edge state wave functions. The Hartree simulations also show how additional deeply bound states in charge accumulation regions close to $\mathrm{QH}$ edges can modify the edge states.

This paper will review the bent quantum well system at both zero magnetic field and at high fields of equal filling factor on both facets. In the first section, the structure will be reviewed and new TEM pictures demonstrating the sharpness of the corner potential will be shown. In the following section, the Schroedinger equation for this system will be solved both with and without magnetic field, detailing the energy eigenstates in both cases. To develop intuition for the resulting dispersion in the low- $B$ limit, a simplified model is introduced based on the 1D Schroedinger equation for the quantum well. The next section shows how the Hartree solutions match closely to a simple quantum Hall edge, provided that a deeply bound quantum wire state is projected out. In the high- $B$ limit, the subsequent section shows the resulting strong hybridization of the states, leading to dispersions with large gaps between hybridized Landau bands and regions of positive and negative curvature as each band traverses the corner. Concluding remarks then address how published experimental results can be interpreted with the microscopic understanding presented here.

\section{TEM AND CORNER SHARPNESS IN THE TRANSPORT STRUCTURE}

The growth technique and complete layer structure are presented in Refs. 15 and 21, with layer thicknesses summarized in Fig. 1(a). The focus of this work is the electronic structure near the corner junction where electrons accumulate. A bent two-dimensional electron system (2DES) forms at the interface between an MBE grown GaAs base layer and an AlGaAs spacer.

The TEM images in Figs. 1(b) and 1(c) show a crosssection of the GaAs/AlGaAs interface at the overgrown corner, demonstrating a sharp corner profile with an effective diameter of curvature of $5.7 \mathrm{~nm}$. The high-contrast stripe along the diagonal in Fig. 1(c) is an accumulation of $\mathrm{Al}$ adatoms, which occurs due to the slower diffusion of Al compared to $\mathrm{Ga}^{21,22}$ While previous high-resolution TEM measurements were restricted to a corner-overgrown test structure with AlAs/GaAs interfaces, ${ }^{21}$ exploiting the increased contrast between AlAs and $\mathrm{GaAs}$, the images presented here show for the first time that the GaAs/AlGaAs interfaces of corner-overgrown transport samples yield corner profiles with similar nanometer-scale sharpness.

The validity of the sharp-corner assumption for Hartree calculations depends on the corner curvature in relation to relevant quantum length scales. ${ }^{21}$ For example, the onedimensional accumulation wire in Fig. 3 is predicted to exist at $B=0$ in the corner-overgrown heterostructures if the diameter of curvature at the corner $2 r$ is smaller than half the Fermi wavelength $\lambda_{F}{ }^{21}$ For typical sheet electron densities between
$1.0 \times 10^{11}$ and $1.5 \times 10^{11} \mathrm{~cm}^{-2}, \lambda_{F}$ is between 80 and $65 \mathrm{~nm}$. With $2 r=5.7 \mathrm{~nm}$, as evident from Fig. 1(c), the condition $2 r<\lambda_{F} / 2$ is therefore satisfied, and a one-dimensional wire with a single occupied subband should exist at the corner.

To calculate the electronic dispersions in the presence of a $B$-field, the comparison between the magnetic length $l_{B}$ and the triangular confinement width $W$ becomes relevant. The wavefunction full width at half maximum $W=18 \mathrm{~nm}$ is estimated from Hartree calculations of the triangular confinement for the above densities. For small magnetic fields such that $W / 2<l_{B}$ ( $B<8 \mathrm{~T})$, the $B=0$ Hartree potential can be safely used in place of the finite $B$ Hartree potential, drastically simplifying the dispersion calculation. At larger magnetic fields such that $W / 2>l_{B}(B>8 \mathrm{~T})$, dispersion calculations should include the $B$ field in the Hartree iteration. Finally, at extreme fields, the radius of curvature of the corner $r_{\mathrm{eff}}=5 \mathrm{~nm}$ becomes important once $r>l_{B}(B>26 \mathrm{~T})$. At these high fields, the sharp corner approximation for the external potential should be replaced with a real potential with finite corner curvature. The corner-overgrown profiles in Fig. 1 are sharp enough to achieve the fractional filling factor $v=1 / 3$ for typical densities before reaching this limit.

\section{HARTREE SIMULATION AT $B=0$}

The Hartree simulation of the bent quantum well is presented first at zero magnetic field, assuming a perfectly symmetric sample with equal spacer thicknesses and donor concentrations on both facets yielding a sheet electron density of $1.0 \times 10^{11} \mathrm{~cm}^{-2}$. The potential and charge density distribution as well as eigenstates and energy eigenvalues for electrons in the heterostructure are obtained from a self-consistent solution to the Schroedinger equation:

$$
\left[\frac{(\vec{p})^{2}}{2 m^{\star}}+\Phi_{\mathrm{cb}}(\vec{r})+\Phi_{\mathrm{el}}(\vec{r})\right] \Psi(\vec{r})=E \Psi(\vec{r}),
$$

and the Poisson equation

$$
\Delta \Phi=-\frac{\varrho}{\epsilon},
$$

where $\vec{r}=(x, y, z)$ is the position vector, $\varrho$ is the charge density, and $\epsilon$ is the low-frequency dielectric constant for AlGaAs or GaAs, respectively. The potential $\Phi_{\mathrm{cb}}$ is defined by the conduction band of the intrinsic GaAs/AlGaAs semiconductor crystal, and $\Phi_{\mathrm{el}}$ is the Hartree potential of the electron distribution. In the following, the total potential $\Phi=\Phi_{\mathrm{cb}}+\Phi_{\mathrm{el}}$ will be referred to as the Hartree potential. The Poisson equation is solved for a finite cross-section of the heterostructure with the boundary conditions

$$
\Phi_{\text {surf }}=0, \quad \overrightarrow{\mathcal{E}}_{\text {sub }}=0,
$$

where the potential $\Phi_{\text {surf }}$ at the sample surface and the electric field $\overrightarrow{\mathcal{E}}_{\text {sub }}$ at the interface to the bulk of the sample are set to zero. This surface potential assumes midgap pinning of the cap layer.

The Hartree potential $\Phi$ obtained from the self-consistent solution of Eqs. (1) and (2) is plotted in Fig. 2 along a perpendicular cross-section through one facet far from the corner as a function of the distance $d$ from the sample surface. The conduction band bending at the GaAs/AlGaAs interface 


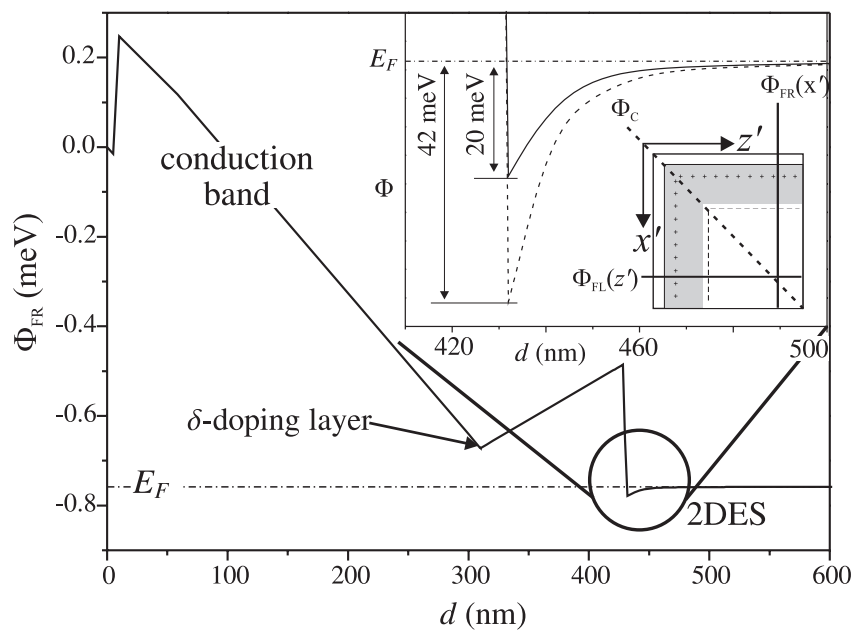

FIG. 2. Self-consistent $B=0$ Hartree potential $\Phi$ for the bent quantum well heterostructure, plotted along a perpendicular crosssection through one of the facets. The two-dimensional electron densities on both facets are $1 \times 10^{11} \mathrm{~cm}^{-2}$. The inset figure shows that the diagonal cross-section through the corner has almost exactly twice the confinement potential as the perpendicular cross-section far from the corner. A 1D accumulation wire results from the additional depth of the potential at the corner.

defines an approximately triangular quantum well which hosts the 2DES in the facets. The inset shows the potential $\Phi_{\mathrm{FL}}\left(z^{\prime}\right)$ $\left[\Phi_{\mathrm{FR}}\left(x^{\prime}\right)\right]$, along a perpendicular cross-section through the left (right) facet far from the corner (solid line), and the potential $\Phi_{c}$ along a diagonal cross-section through the corner junction (dotted line). Due to the confinement in both $x^{\prime}$ and $z^{\prime}$ directions the corner potential $\Phi_{c}$ has about twice the binding potential compared to the facets. The additional depth of the potential well at the corner causes the $1 \mathrm{D}$ accumulation with a binding energy of $7 \mathrm{meV}$, compared to $3.5 \mathrm{meV}$ for electrons in the $2 \mathrm{D}$ facets.

Figure 3(b) shows the electron density calculated at $B=$ 0 , integrated over the quantum well thickness and projected onto the $x$ axis indicated in Fig. 3(a). We distinguish three different electron systems in the corner region: the 2D systems in the facets plotted in red or blue, respectively, and a 1D accumulation wire plotted in green. Figure 3(c) shows the calculated binding energies relative to the Fermi level $E_{F}$ for $2 \mathrm{D}$ and 1D states in the bent quantum well, where the 1D wire is approximately twice as deeply bound as the 2D ground energies, due to confinement in $z^{\prime}$ and $x^{\prime}$ directions. Note that the discrete spectrum of 2D states is an artifact of the finite size of the quantum-mechanical simulation.

\section{HARTREE SIMULATION AT FINITE $B$}

Having identified the 2D and 1D states that exist in a bent quantum well at zero magnetic field, we now study the new states that emerge from these subsystems at finite $B$. If a tilted magnetic field is applied such that the $2 \mathrm{D}$ electron systems of both facets are in a quantum Hall state, all three subsystems are strongly coupled and form the bent quantum Hall system. In this paper, we restrict ourselves to $\mathrm{BQH}$ systems where the magnetic field is applied at $4^{\circ}$ angle such that the $2 \mathrm{D}$ systems on both facets are in the same quantum Hall state with filling
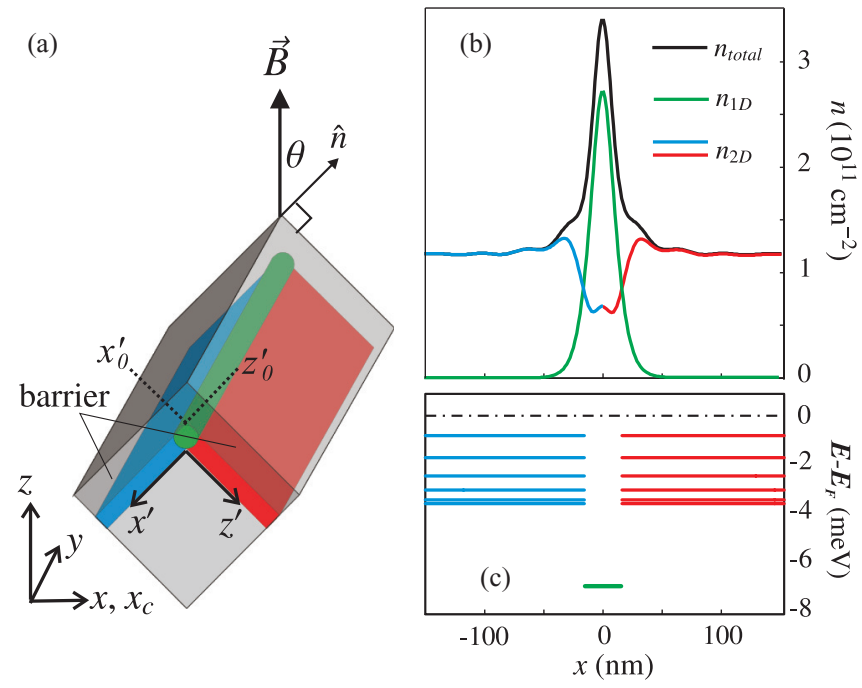

FIG. 3. (Color online) (a) Two coordinate frames defined for the Hartree simulations at finite $B$. The $(x, y, z)$ frame is used for the momentum and cyclotron orbit center coordinates, with the $z$ axis parallel to $\vec{B}$. The $\left(x^{\prime}, z^{\prime}\right)$ coordinate frame fixed to the sample is convenient to plot the electronic wave functions in real space. The plots in (b) show the calculated electron densities projected onto the $x$ axis indicated in the schematic, where the blue and red curves are the $2 \mathrm{D}$ electron densities in the facets and the blue and red lines at the bottom represent eigenenergies of states in the facets. The green curve is the density of the $1 \mathrm{D}$ accumulation wire with eigenenergy represented by the green line at the bottom of the figure. The black curve shows the total electron density. (c) The calculated binding energies for the 2D and 1D states.

factor $v$. Zeeman spin splitting and exchange interactions are neglected. It is convenient to pick a coordinate frame $(x, y, z)$, where the $z$ axis is defined by the magnetic field and the $y$ axis is the translationally invariant direction along the corner of the bent quantum well, as shown in Fig. 3(a). We choose the vector potential $\vec{A}$ in the Landau gauge, which preserves $y$-translational invariance and makes the momentum $k_{y}$ a good first quantum number:

$$
\vec{A}=x B \hat{y}
$$

which satisfies the condition

$$
\vec{B}=\vec{\nabla} \times \vec{A}=B \hat{z} .
$$

As an ansatz for the wave functions, we choose

$$
\Psi(\vec{r})=\psi_{n, k_{y}}(x, z) e^{i k_{y} y},
$$

where $n$ is the orbital quantum number. Only the lowest electrostatically confined subband is occupied in these heterostructures. With the ansatz (5) the explicitly $y$-dependent parts can be separated from the Schroedinger equation

$$
\left[\frac{(\vec{p}-e \vec{A})^{2}}{2 m^{\star}}+\Phi(x, z)\right] \Psi(\vec{r})=E \Psi(\vec{r}),
$$

and one obtains

$$
\begin{aligned}
& {\left[\frac{\left(p_{x}^{2}+p_{z}^{2}\right)}{2 m^{\star}}+\frac{1}{2} m^{\star} \omega_{c}^{2}\left(x-x_{c}\right)^{2}+\Phi(x, z)\right] \psi_{n, k_{y}}(x, z)} \\
& =E_{n, k_{y}} \psi_{n, k_{y}}(x, z) .
\end{aligned}
$$


We see that the magnetic field causes the additional harmonic potential $\frac{1}{2} m^{\star} \omega_{c}^{2}\left(x-x_{c}\right)^{2}$ in the $x$ direction perpendicular to $\vec{B}$, where $l_{B}=\sqrt{\frac{\hbar}{e B}}$ is the magnetic length and $\omega_{c}=\frac{e B}{m^{\star}}$ is the cyclotron frequency. The cyclotron orbit center $x_{c}$ depends linearly on the momentum in $y$ direction: $x_{c}=k_{y} l_{B}^{2}$. This correspondence between the momentum $k_{y}$ and the orbit center position $x_{c}$ allows one to project a real-space picture of the dispersion $E_{n}\left(k_{y}\right)=E_{n}\left(x_{c}\right)$ in orbit center coordinates.

\section{A. Hybrid dispersion}

Figure 4(a) shows the dispersion calculated for a bent quantum well with sheet electron densities $n=1.0 \times 10^{11} \mathrm{~cm}^{-2}$ in both facets, where a magnetic field of $1.5 \mathrm{~T}$ applied at a tilt angle $\theta=45^{\circ}$ relative to the facet normal vectors brings both $2 \mathrm{D}$ systems to the $v=4$ quantum Hall state. The dispersion of this bent quantum Hall system is plotted in black as binding
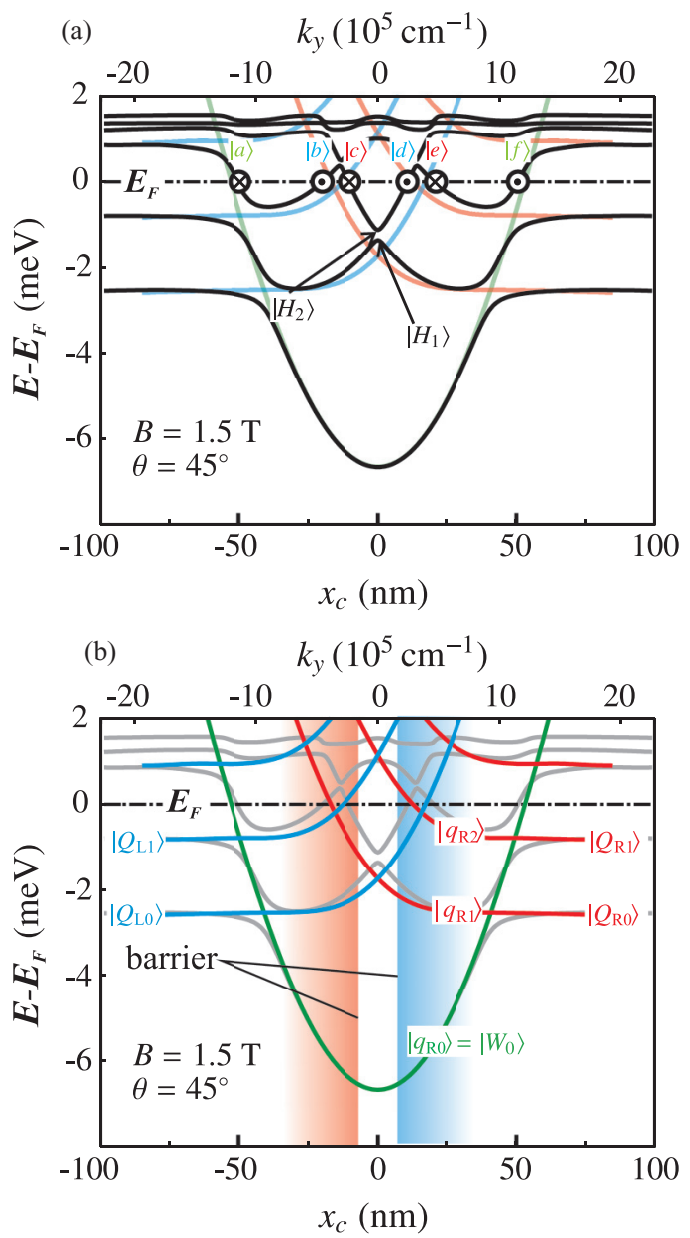

FIG. 4. (Color online) (a) Hartree dispersion (black) of the bent quantum Hall system calculated for a magnetic field of $1.5 \mathrm{~T}$ at a tilt angle $\theta=45^{\circ}$, plotted in as binding energies $E$ vs cyclotron orbit center $x_{c}$ or momentum $k_{y}$, respectively. (b) shows only the idealized sharp QH edge dispersions of the right-facing (blue) and left-facing (red) facets and the parabolic dispersion of the 1D wire (green). The dotted lines and shaded areas indicate the position of the effective hard walls seen by the edge states. For comparison, the dispersion of (b) is plotted in the background of (a) and vice versa. Wave functions for each branch are labeled according to the discussion in Sec. IV. energy $E$ versus the momentum $k_{y}$ (top axis) and the orbit center $x_{c}$ (bottom axis).

To provide intuition as to the origin of these states, we define a constituent system of the three separate systems that hybridize in the bent quantum well: the left facet quantum well, the right facet quantum well, and the 1D accumulation wire at the corner.

The potentials $\Phi_{\mathrm{QL}}\left(x^{\prime}, z^{\prime}\right)$ and $\Phi_{\mathrm{QR}}\left(x^{\prime}, z^{\prime}\right)$ of the left and right facet quantum wells are modeled with the single-facet confinement potentials $\Phi_{\mathrm{FL}}\left(z^{\prime}\right)$ and $\Phi_{\mathrm{FR}}\left(x^{\prime}\right)$ shown in the inset of Fig. 2 (solid line plot), terminated by a sharp barrier of $0.3 \mathrm{eV}$ height at the position $x_{0}^{\prime}$ or $z_{0}^{\prime}$, respectively, as indicated in Fig. 3:

$$
\begin{aligned}
& \Phi_{\mathrm{QL}}\left(x^{\prime}, z^{\prime}\right)= \begin{cases}\Phi_{B}, & x^{\prime}<x_{0}^{\prime}, \\
\Phi_{\mathrm{FL}}\left(z^{\prime}\right), & x^{\prime}>x_{0}^{\prime},\end{cases} \\
& \Phi_{\mathrm{QR}}\left(x^{\prime}, z^{\prime}\right)= \begin{cases}\Phi_{B}, & z^{\prime}<z_{0}^{\prime}, \\
\Phi_{\mathrm{FR}}\left(x^{\prime}\right), & z^{\prime}>z_{0}^{\prime},\end{cases}
\end{aligned}
$$

where $\Phi_{B}=E_{F}+0.3 \mathrm{eV}$. The dispersion calculated for the right-facing quantum well is shown in blue in Fig. 4(b) for the barrier positioned at the vertical blue line, mirrored by the red dispersion of the left-facing quantum well. At $B=1.5 \mathrm{~T}$, these quasiparabolic dispersions represent the edge states at a $v=4$ sharp quantum Hall edge. The confinement potential $\Phi_{W}\left(x^{\prime}, z^{\prime}\right)$ of the $1 \mathrm{D}$ accumulation wire is modeled as the product

$$
\Phi_{W}\left(x^{\prime}, z^{\prime}\right)=\Phi_{\mathrm{FR}}\left(x^{\prime}\right) \times \Phi_{\mathrm{FL}}\left(z^{\prime}\right) .
$$

The deeply bound accumulation wire is indicated by the green parabolic dispersion in Fig. 3. The dispersions of these constituent systems in Fig. 4(b) clearly represent the key features of the total Hartree dispersion.

The dispersion of the bent quantum Hall system (black) in Fig. 4(a) can now be discussed in terms of the constituent left facet, right facet, and accumulation wire dispersions shown in Fig. 4(b). Even though there is no tunnel barrier separating the two quantum wells of the BQH system, we identify the two hard-wall-like dispersions of the $\mathrm{QH}$ edges from both facets, which arise because the sudden $90^{\circ}$ bend in the heterojunction serves as a hard wall for the incident skipping orbits within the opposing facet, and counterpropagating edge states from the two orthogonal facets interpenetrate at the corner. The total dispersion of the bent quantum Hall system emerges as the three subsystem dispersions anticross, and a 1D hybrid system forms along the corner. The coupling between states opens up anticrossing gaps where the dispersions cross, and states with larger spatial overlap (such as the wire and facet states) have larger coupling and larger gaps, whereas states with less spatial overlap (such as between opposing facets) have very small gaps.

Conducting modes are found at the intersection points of the dispersion curves with the Fermi level $E_{F}$ in Fig. 4(a). The direction of propagation is determined by the slope of the dispersion at the intersection, where a positive slope leads to a forward-propagating mode and a negative slope yields a reverse-propagating mode, as indicated by the different symbols in Fig. 4(a), where the blue edge states are forwardpropagating and the red edge states reverse-propagating. At 


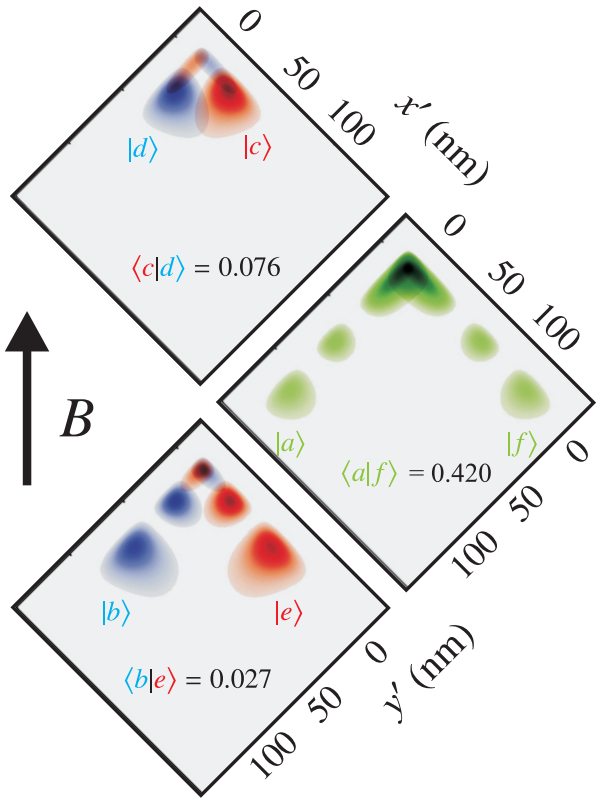

FIG. 5. (Color online) Color-map plots of the probability amplitudes for real-space wave functions corresponding to the various $1 \mathrm{D}$ modes marked in Fig. 4(a). The electrons in the facets (blue and red) and corner accumulation wire (green) are colored according to their Hartree dispersions shown in Fig. 4(b).

low magnetic fields, for example, at filling factor $v=4$ in Fig. 4(a), the Hartree simulations show $v=4$ spin-degenerate conducting modes from each quantum Hall edge. The 1D accumulation wire adds two spin-degenerate modes in each direction and serves as an additional 1D channel for scattering. Together with the 1D edge modes from the QH systems, the model thus predicts as many as $N=v+2$ one-dimensional modes in each direction. Fig. 5 shows the real-space wave functions of the 1D modes marked in Fig. 4(a). The sharp edge potential prohibits the formation of compressible and incompressible strips, ${ }^{23}$ which allows for a finite overlap of the wave functions corresponding to the conducting modes, as shown in Fig. 5.

\section{B. Hybrid wave functions}

In this section, we show how the hybrid eigenstates at the anticrossing points of the dispersion in Fig. 4 can be described in terms of the wave functions of the planar constituent systems. These considerations help to develop a general intuition for understanding the eigenstates that emerge when various quantum mechanical systems, here a $1 \mathrm{D}$ wire and two planar quantum Hall systems with sharp edges, are coupled at a nonplanar junction.

We will define hybrid wave functions $\left|q_{L}\right\rangle$ or $\left|q_{R}\right\rangle$ in terms of the constituent wave functions $\left|Q_{L}\right\rangle$ or $\left|Q_{R}\right\rangle$ of the left or right quantum well, respectively, and $|W\rangle$ in the wire. To address this, we calculate the eigenstates $\left|Q_{L m, x_{c}}\right\rangle$ or $\left|Q_{R m, x_{c}}\right\rangle$ of the sharp quantum Hall edge potentials $\Phi_{\mathrm{QL}}\left(x^{\prime}, z^{\prime}\right)$ or $\Phi_{\mathrm{QR}}\left(x^{\prime}, z^{\prime}\right)$, respectively, where $m$ is the Landau level index. Then the accumulation wire state $\left|W_{0, x_{c}}\right\rangle$ is calculated as $\left|q_{L 0, x_{c}}\right\rangle=\left|q_{R 0, x_{c}}\right\rangle=\left|W_{0, x_{c}}\right\rangle$. In the following, we restrict ourselves to wave functions of the right-facet quantum Hall system. The left-facet states are constructed analogously. At orbit centers away from anticrossings of the QH edge and 1D wire dispersions, for example, at $x_{c}=22 \mathrm{~nm}$ in Fig. 4, the higher energy eigenstates $\left|q_{R n, x_{c}}\right\rangle$ for the combined system are subsequently constructed by simply projecting out the $1 \mathrm{D}$ wire state and all the previously constructed states with quantum numbers $i<n$ from the $\mathrm{QH}$ edge states $\left|Q_{R m=n-1, x_{c}}\right\rangle$ :

$$
\begin{gathered}
\left|q_{R 0, x_{c}}\right\rangle=\left|W_{0, x_{c}}\right\rangle, \\
\left|q_{R 1, x_{c}}\right\rangle=N_{1, x_{c}}\left\{\left|Q_{R 0, x_{c}}\right\rangle-\left|q_{R 0, x_{c}}\right\rangle\left\langle q_{R 0, x_{c}} \mid Q_{R 0, x_{c}}\right\rangle\right\}, \\
\left|q_{R 2, x_{c}}\right\rangle=N_{2, x_{c}}\left\{\left|Q_{R 1, x_{c}}\right\rangle-\left[\left|q_{R 0, x_{c}}\right\rangle\left\langle q_{R 0, x_{c}}\right|\right.\right. \\
\left.\left.\left.+\left|q_{R 1, x_{c}}\right\rangle\left\langle q_{R 1, x_{c}}\right|\right] Q_{R 1, x_{c}}\right\rangle\right\}, \\
\left|q_{R n, x_{c}}\right\rangle=N_{n, x_{c}}\left\{\mathbb{1}-\sum_{i<n}\left|q_{R i, x_{c}}\right\rangle\left|q_{R i, x_{c}}\right|\right\}\left|Q_{R m=n-1, x_{c}}\right\rangle,
\end{gathered}
$$

where $N_{n, x_{c}}$ are normalizing constants. This construction ensures that the states $\left|q_{R n, x_{c}}\right\rangle$ are orthogonal to all states $\left|q_{R i<n, x_{c}}\right\rangle$, and therefore form a new orthonormal basis of eigenstates.

Figure 6(a) shows one-dimensional cross-sections along the $z^{\prime}$ axis at $x^{\prime}=0$ [center of the red quantum well in Fig. 4(a)] of the original wave functions $\left|Q_{R m, x_{c}}\right\rangle$ and $\left|W_{0, x_{c}}\right\rangle$ [see Fig. 4(b)]. The hard wall indicated by the vertical dotted line is positioned at $z^{\prime}=-10 \mathrm{~nm}$ and the $B$-field induced harmonic potential $\Phi_{B}$ (gray curve) is centered at $x_{c}=22 \mathrm{~nm}$. Note that the harmonic potential from Eq. (8) is projected onto the $z^{\prime}$ axis in Fig. 4(a), where $z^{\prime}=31 \mathrm{~nm}$.

To demonstrate that the full two-dimensional cross-section of the bent quantum Hall system can be closely approximated in terms of the new basis states $\left|q_{R n, x_{c}}\right\rangle$, we construct the hybrid states $\left|h_{n, x_{c}}\right\rangle$ at the $x_{c}=0$ anticrossing in Fig. 4(a), using symmetric and antisymmetric combinations of the wave

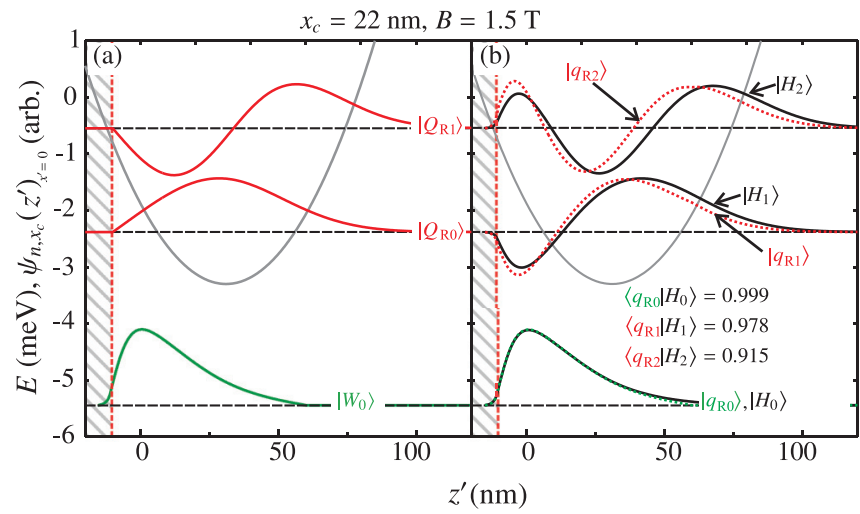

FIG. 6. (Color online) Cross-sections along the $z^{\prime}$ axis at $x^{\prime}=0$ of wave functions for the single quantum Hall edge states $\left|Q_{R m}\right\rangle$ and the $1 \mathrm{D}$ accumulation wire state $\left|W_{0}\right\rangle$ at $B=1.5 \mathrm{~T}$ and $x_{c}=$ $22 \mathrm{~nm}$ are shown in (a). The wave functions $\left|q_{R n}\right\rangle$, plotted in (b), are constructed after Eqs. (11)-(14) and show excellent agreement with the Hartree eigenstates $\left|H_{n}\right\rangle$ at $x_{c}=22 \mathrm{~nm}$ obtained from the numerical simulation of the whole bent quantum Hall system. 


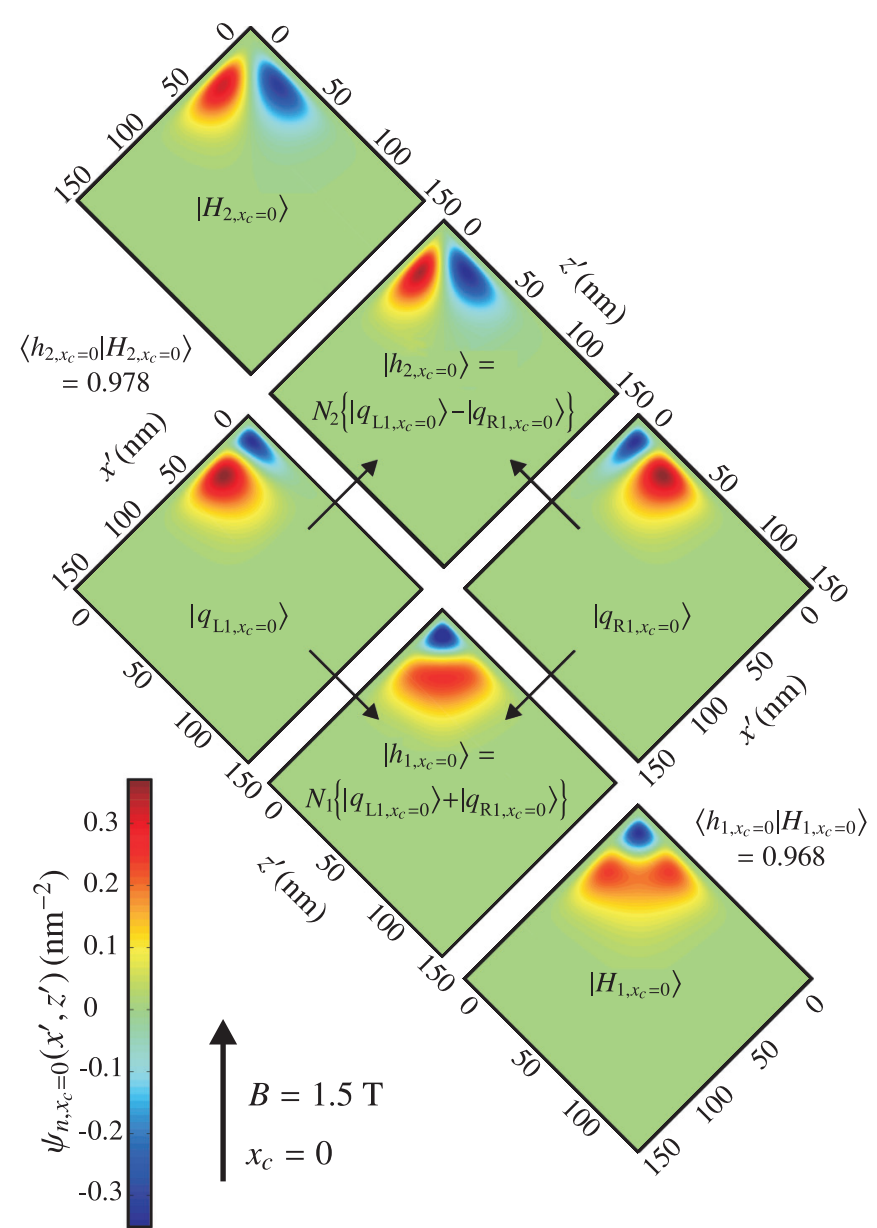

FIG. 7. (Color online) Color map plots illustrating the construction of binding $\left(\left|h_{1, x_{c}=0}\right\rangle\right)$ and antibinding states $\left(\left|h_{2, x_{c}=0}\right\rangle\right)$ in a bent quantum Hall system at $x_{c}=0$. The constructed wave functions agree very well with the numerical Hartree results $\left(\left|H_{1, x_{c}=0}\right\rangle\right.$ and $\left.\left|H_{2, x_{c}=0}\right\rangle\right)$, with an overlap of $97 \%-98 \%$.

functions $\left|q_{L n, x_{c}}\right\rangle$ and $\left|q_{R n, x_{c}}\right\rangle$ of the left and right quantum Hall edges:

$$
\begin{gathered}
\left|h_{1, x_{c}=0}\right\rangle=N_{1}\left\{\left|q_{L 1, x_{c}=0}\right\rangle+\left|q_{R 1, x_{c}=0}\right\rangle\right\}, \\
\left|h_{2, x_{c}=0}\right\rangle=N_{2}\left\{\left|q_{L 1, x_{c}=0}\right\rangle-\left|q_{R 1, x_{c}=0}\right\rangle\right\},
\end{gathered}
$$

where $N_{1}$ and $N_{2}$ are normalizing constants. Figure 7 shows color map plots of the wave functions $\psi_{n, x_{c}=0}\left(x^{\prime}, z^{\prime}\right)$ corresponding to states at the $x_{c}=0$ anticrossing of the left and right quantum Hall edges. The states $\left|h_{1, x_{c}=0}\right\rangle$ and $\left|h_{2, x_{c}=0}\right\rangle$ constructed after Eqs. (15) and (16) show excellent agreement with the numerical results $\left|H_{1, x_{c}=0}\right\rangle$ and $\left|H_{2, x_{c}=0}\right\rangle$ for the binding or antibinding states at the $x_{c}=0$ anticrossing gap, respectively.

\section{The high $B$-field limit}

Figure 8 shows Hartree dispersions of the bent QH system at various magnetic fields. With increasing $B$ the anticrossing gaps in the dispersion increase, indicating an increasing coupling among the three constituent subsystems. This follows from the reduced magnetic length, which brings all quantum confined 1D states closer to the corner, and therefore

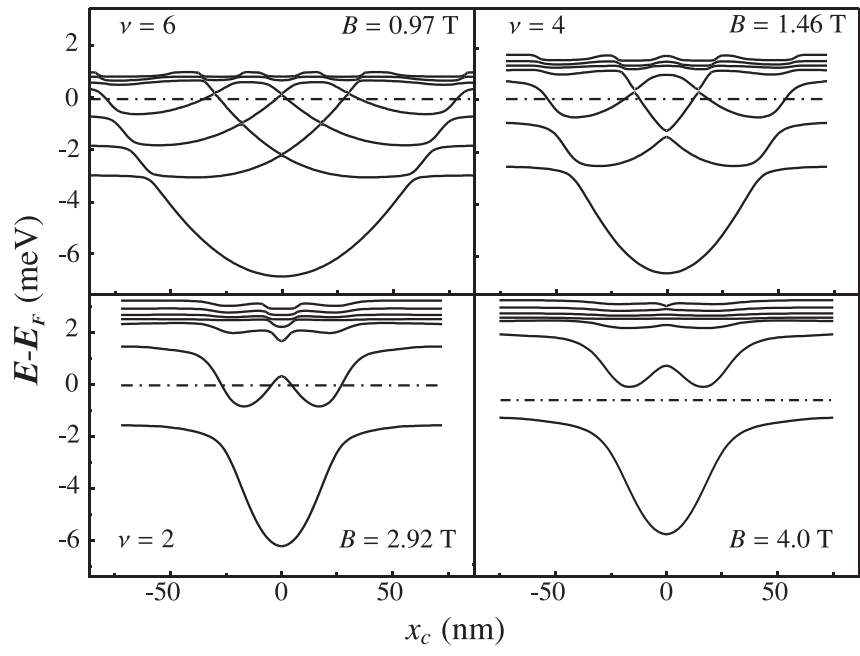

FIG. 8. Hartree dispersions of a bent quantum Hall junction at various magnetic fields. In contrast to planar barrier systems where the gaps vanish exponentially at high $B$, here the anticrossing gaps due to strong coupling of the modes at the corner increase with increasing magnetic field.

in stronger overlap with each other. This is in contrast to planar barrier systems, for example, the planar antiwire of Refs. 24 and 25 , where the coupling decreases exponentially at high $B$. The strong anticrossings result in both positive curvatures corresponding to an electron-like mass and negative curvatures representing a hole-like mass in parts of the dispersion, and the band gaps between hybridized Landau bands start to become larger than the bandwidths of the higher excited bands. The essential feature of a bound wire state at the corner evolves into a local dispersion minimum for the lowest band.

\section{INTERPRETATION OF EXPERIMENTS}

A series of transport experiments have already been performed on bent quantum Hall systems. Conductance measurements along the $\mathrm{BQH}$ junction have shown weakly insulating, strongly insulating, and metallic behavior, depending on $v .{ }^{16}$ At low magnetic fields $(v=3,4,5,6)$, weakly insulating behavior was observed, with a finite, yet nonuniversal conductance of a few percent of the quantized value $v e^{2} / h$ and little temperature or dc voltage bias dependence. In contrast, the conductance at higher magnetic fields $(v=1,2)$ showed strongly insulating behavior and decreased drastically with decreasing temperature or voltage. Finally, at the fractional filling factor $v=1 / 3$, the $\mathrm{BQH}$ junction showed metallic behavior, where the conductance strongly increased upon lowering the temperature or voltage. Possible explanations for the unique transport properties of the bent quantum Hall junction, should be consistent with the experimentally measured sharp junction curvature in Fig. 1 and the basic results of the Hartree simulations.

A 1D multimode conductor, as shown in the $v=4$ simulation in Fig. 4, could be causing the weakly insulating behavior of the bent quantum Hall junction observed at low magnetic fields. A finite overlap of wave functions corresponding to the conducting modes (see Fig. 5) becomes possible due to the suppression of incompressible strips ${ }^{23}$ 
and the absence of a barrier at the sharp corner. Therefore disorder-induced scattering among various modes can lead to diffusive conduction with a nonuniversal conductance in this multimode $1 \mathrm{D}$ conductor.

The Hartree simulations in the high- $B$ limit in Fig. 8 show increasing anticrossing gaps due to a stronger coupling among the three constituent subsystems with increasing $B$. The increased gaps are likely to form a band insulator at high magnetic fields, which is one possible explanation for the strongly insulating behavior of the $\mathrm{BQH}$ junction at filling factors $v=1,2$. However, the experimental results in Refs. 16 and 17 apparently favor a localization transition leading to the insulating behavior.

At the extreme magnetic fields of approximately $23 \mathrm{~T}$ needed to reach $v=1 / 3$ in both facets, ${ }^{16}$ the Hartree dispersions from Eq. (1) would have to be calculated self-consistently at finite $B$, and must include interactions to correctly account for the Laughlin ground state in the facets. Though such calculations are beyond the scope of this work, one qualitatively expects a mixing of the accumulation wire dispersion for electrons with the fractional $\mathrm{QH}$ edge dispersions for quasiparticles, ${ }^{26}$ where the resulting states could possibly be approximated by a method similar to the construction described in Eqs. (11)-(16). Even without calculating the exact form for the dispersion, based on the simulations at lower $B$ we can predict that it will have a local minimum at the corner, and that due to the strong coupling, the hybridized gaps will be large in comparison to the intersubband gaps. As discussed in Refs. 16,19, and 20, coupled fractional QH edges can result in the observed metallic behavior, as long as electrons (not fractional quasiparticles) backscatter the charge between the counterpropagating $v=1 / 3$ edges, creating an "antiwire." Prerequisites are a sufficiently strong coupling of counterpropagating edge modes, and an effective "vacuum" for the fractional quasiparticles, to ensure electron scattering. The Hartree simulations presented in this paper, though restricted to the integer $\mathrm{QH}$ regime, infer that both conditions are fulfilled in the bent quantum Hall system at hand, where the dispersions shown in Fig. 8 indicate an increasing coupling of counterpropagating edge states, and the 1D accumulation wire in Fig. 4 could function as a $v=1$ "vacuum" for the $v=1 / 3$ quasiparticles. This situation is in contrast to the scenario of the planar antiwire geometry originally suggested in Refs. 19 and 20 and implemented in Refs. 24 and 25, where the tunnel barrier separating the two coplanar QH systems exponentially suppresses tunneling at high $B$, thereby prohibiting the desired strong coupling of fractional $\mathrm{QH}$ edges. We thus propose that, at the high magnetic fields necessary to see $v=1 / 3$ edges, the bent quantum Hall system, where the corner geometry allows the strong coupling of two $v=1 / 3$ edges without a separating tunnel barrier, is the only experimentally realizable system that can show the predicted "antiwire" system of Refs. 19 and 20.

\section{CONCLUSION}

We have studied a new type of low-dimensional system, the bent quantum Hall junction, in spin-degenerate Hartree simulations, providing an elementary understanding of the various coupled electronic states existing in such a nonplanar junction of two quantum Hall systems. We have shown how the electronic dispersion and eigenstates in this hybrid system can still be quantitatively understood in terms of the dispersion and eigenstates of constituent QW facet and accumulation wire subsystems. Such analysis can simplify future simulations: instead of a fully numerical solution to the Schroedinger equation one could solve the 1D Hartree potential of a single quantum well, and then construct the dispersion and eigenstates from template wave functions of the subsystem states. With such a semianalytical approach the simulations could also be extended to allow for self-consistent simulations at finite magnetic fields or to approximate the hybridized fractional $\mathrm{QH}$ states expected in the $\mathrm{BQH}$ junction at high $B$. The simulations clearly demonstrate how the bent quantum Hall system differs from any tunnel-coupled planar system of counterpropagating $\mathrm{QH}$ edges. Particularly, the increasing wave function overlap of the edge states with increasing $B$ makes it an interesting device to study coupled fractional quantum Hall edges, and possibly the only system where the predicted 1D metallic behavior at $v=1 / 3$ can be observed experimentally.

\section{ACKNOWLEDGMENTS}

This work was supported by the Deutsche Forschungsgemeinschaft in the Schwerpunktprogramm Quanten Hall Systeme, by DFG GR 2618/1-1, by NSF CAREER Award DMR-0748856, the Sonderforschungsbereich SFB 621, and the Nanosystems Initiative Munich.
*Current address: Center for Functional Nanomaterials, Brookhaven National Laboratory, Upton, NY 11973, USA.

${ }^{\dagger}$ Current address: Physics Department, Universität Regensburg, D-93053, Germany.

${ }^{\ddagger}$ Corresponding author: m-grayson@ northwestern.edu

${ }^{1}$ K. S. Novoselov, A. K. Geim, S. V. Morozov, D. Jiang, Y. Zhang, S. V. Dubonos, I. V. Grigorieva, and A. A. Firsov, Science 306, 666 (2004).

${ }^{2}$ S. Das Sarma, S. Adam, E. H. Hwang, and E. Rossi, Rev. Mod. Phys. 83, 407 (2011).

${ }^{3}$ S. Iijima, Nature (London) 354, 56 (1991).

${ }^{4}$ T. Ando, A. B. Fowler, and F. Stern, Rev. Mod. Phys. 54, 437 (1982).
${ }^{5}$ K. v. Klitzing, G. Dorda, and M. Pepper, Phys. Rev. Lett. 45, 494 (1980).

${ }^{6}$ D. C. Tsui, H. L. Stormer, and A. C. Gossard, Phys. Rev. Lett. 48, 1559 (1982).

${ }^{7}$ M. Grayson, D. C. Tsui, L. N. Pfeiffer, K. W. West, and A. M. Chang, Phys. Rev. Lett. 86, 2645 (2001).

${ }^{8}$ Yi Cui, X. Duan, J. Hu, and C. M. Lieber, J. Phys. Chem. B 104, 5213 (2000).

${ }^{9}$ Giulio Ferrari, Andrea Bertoni, Guido Goldoni, and Elisa Molinari, Phys. Rev. B 78, 115326 (2008).

${ }^{10}$ M. Heigoldt et al., J. Mater. Chemistry 19, 840 (2009).

${ }^{11}$ M. Fickenscher et al., Nano Lett. 13, 1016 (2013). 
${ }^{12}$ M. Grayson, C. Kurdak, D. C. Tsui, S. Parihar, S. Lyon, and M. Shayegan, Solid State Electron. 40, 233 (1996).

${ }^{13}$ A. Yacoby, H. L. Stormer, K. W. Baldwin, L. N. Pfeiffer, and K. W. West, Solid State Commun. 101, 77 (1997); R. de Picciotto, H. L. Stormer, A. Yacoby, L. N. Pfeiffer, K. W. Baldwin, and K. W. West, Phys. Rev. Lett. 85, 1730 (2000).

${ }^{14}$ J. Moser, T. Zibold, D. Schuh, M. Bichler, F. Ertl, G. Abstreiter, M. Grayson, S. Roddaro, and V. Pellegrini, Appl. Phys. Lett. 87, 052101 (2005); J. Moser, S. Roddaro, D. Schuh, M. Bichler, V. Pellegrini, and M. Grayson, Phys. Rev. B 74, 193307 (2006).

${ }^{15}$ M. Grayson, D. Schuh, M. Huber, M. Bichler, and G. Abstreiter, Appl. Phys. Lett. 86, 032101 (2005); D. Schuh, M. Grayson, M. Bichler, and G. Abstreiter, Physica E 23, 293 (2004).

${ }^{16}$ M. Grayson, L. Steinke, D. Schuh, L. Hoeppel, J. Smet, D. Maude, K. v. Klitzing, M. Bichler and G. Abstreiter, Phys. Rev. B 76, 201304(R) (2007); L. Steinke, D. Schuh, M. Bichler, G. Abstreiter, and M. Grayson, ibid. 77, 235319 (2008).
${ }^{17}$ L. Steinke, Ph.D. Thesis, Technische Universität München, 2009.

${ }^{18}$ M. Grayson, D. Schuh, M. Bichler, M. Huber, G. Abstreiter, L. Hoeppel, J. Smet, and K. von Klitzing, Physica E 22, 181 (2004).

${ }^{19}$ C. L. Kane and M. P. A. Fisher, Phys. Rev. B 46, 15233 (1992).

${ }^{20}$ S. R. Renn and D. P. Arovas, Phys. Rev. B 51, 16832 (1995).

${ }^{21}$ L. Steinke, P. Cantwell, D. Zakharov, E. Stach, N. J. Zaluzec, A. Fontcuberta i Morral, M. Bichler, G. Abstreiter, and M. Grayson, Appl. Phys. Lett. 93, 193117 (2008).

${ }^{22}$ M. Heiss et al., Nature Materials (2013), doi:10.1038/nmat3557.

${ }^{23}$ M. Huber, M. Grayson, M. Rother, W. Biberacher, W. Wegscheider, and G. Abstreiter, Phys. Rev. Lett. 94, 016805 (2005).

${ }^{24}$ W. Kang, H. L. Stormer, L. N. Pfeiffer, K. W. Baldwin, and K. W. West, Nature (London) 403, 59 (2000).

${ }^{25}$ M. Habl, M. Reinwald, W. Wegscheider, M. Bichler, and G. Abstreiter, Phys. Rev. B 73, 205305 (2006).

${ }^{26}$ X. G. Wen, Phys. Rev. Lett. 64, 2206 (1990). 